\title{
Models to Evaluate the Role of Stress in Periodontal Disease
}

\author{
Robert J. Genco, ${ }^{*}$ Alex W. Ho, ${ }^{*}$ Jeffrey Kopman,* Sara G. Grossi,, Robert G. Dunford,* \\ and Lisa A. Tedesco $\ddagger$ \\ *Department of Oral Biology, Periodontal Disease Research Center, School of Dental Medicine, \\ State University of New York at Buffalo. \\ †Department of Microbiology, School of Medicine and Biomedical Sciences. \\ $¥$ School of Dentistry, University of Michigan, Ann Arbor, MI.
}

\begin{abstract}
We evaluated the association of stress, distress, and coping behaviors with periodontal disease in 1,426 subjects, aged 25 to 74, in Erie County, NY. Demographic characteristics, medical and dental history, and tobacco and alcohol consumption, as well as clinical assessments of supragingival plaque, subgingival flora, gingival bleeding, calculus, probing depth, clinical attachment level $(C A L)$, and radiographic alveolar bone loss $(A B L)$ were obtained for each subject. Subjects also completed a set of 5 psychosocial instruments that measured life events, daily strains, hassles and uplifts, distress, and coping behaviors. Internal consistencies of all subscales on the instruments were high, with Cronbach's alpha ranging from 0.88 to 0.99 . Logistic regression indicated that financial strain was significantly associated with greater attachment and alveolar bone loss (OR 1.70; 95\% CI, 1.09-2.65; and 1.68; 95\% CI, 1.20-2.37, respectively) after adjusting for age, gender, and smoking. When those with financial strain were stratified with respect to coping behaviors, it was found that those who exhibited high emotion-focused coping (inadequate coping) had an even higher risk of having more severe attachment loss (OR 2.24; 95\% CI, 1.15-4.38) and alveolar bone loss (OR 1.91; 95\% CI, 1.15-3.17) than those with low levels of financial strain within the same coping group, after adjustment for age, gender, and cigarette smoking. After further adjusting for number of visits to
\end{abstract}

the dentist, those with financial strain who were high emotion-focused copers still had higher levels of periodontal disease based on CAL (OR 2.12; 95\% CI, 1.07-4.18). In contrast, subjects with high levels of financial strain who reported high levels of problembased coping (good coping) had no more periodontal disease than those with low levels of financial strain. Salivary cortisol levels were higher in a test group exhibiting severe periodontitis, a high level of financial strain, and high emotion-focused coping, as compared to a control group consisting of those with little or no periodontal disease, low financial strain, and low levels of emotionfocused coping (11.04 \pm 4.4 vs. $8.6 \pm 4.1$ $\mathrm{nmol} / \mathrm{L}$ salivary cortisol, respectively). These findings suggest that psychosocial measures of stress associated with financial strain are significant risk indicators for periodontal disease in adults. Further prospective studies are needed to help establish the time course of stress, distress, and inadequate coping on the onset and progression of periodontal disease, as well as to evaluate the mechanisms by which stress exerts its effects on periodontal infections. Ann Periodontol 1998;3: 288-302.

Key Words: Periodontal diseases/pathogenesis; risk factors; stress; coping behavior; saliva/analysis; hydrocortisone. 


\section{INTRODUCTION}

Periodontal diseases are infections associated with specific pathogenic bacteria that colonize the subgingival area. At least two of these microorganisms, Porphyromonas gingivalis and Actinobacillus actinomycetemcomitans, express potent virulence mechanisms and can invade the gingiva. However, as with many chronic infections, the onset and progression of periodontal infections are clearly modified by local and systemic host conditions, or risk factors, that markedly affect the resistance of the host to infecting periodontal organisms.

Systemic risk factors recently identified in large epidemiological studies include diabetes mellitus and cigarette smoking. ${ }^{1-3}$ In addition, gender (being male) and age are associated with increased prevalence and severity of periodontal disease. ${ }^{4}$ It has also been shown that those with acquired immunodeficiency syndrome (AIDS) have periodontal disease, including severe and painful necrotizing forms. ${ }^{5,6}$ Gender, diabetes mellitus, and smoking were associated with more severe periodontal disease after adjusting for possible confounding variables including local factors in the Erie County epidemiological study, using attachment loss ${ }^{2}$ or alveolar bone loss ${ }^{3}$ as the primary outcome variable to measure periodontal disease.

Potential risk factors include osteopenia ${ }^{7}$ and neutrophil disorders. ${ }^{8}$ Genetic polymorphisms may be associated with periodontal disease. For example, the allelic polymorphism of the receptor for IgG2 (FC $\gamma$ RII or CD32) has been proposed to be a risk marker for localized juvenile periodontitis. ${ }^{9}$ In adult periodontitis, the genetic polymorphisms in genes for IL-1 have been associated with higher prevalence and more severe forms of periodontal disease as compared to the moderate forms among non-smokers. ${ }^{10}$

Studies by Grossi et al. ${ }^{2}$ show that selfreported anemia and allergy represent an apparent protective modifier for periodontal disease expression. The mechanisms for these two are unknown; however, they may be related to the therapy provided for these conditions.

Hence, a considerable body of data exists relating systemic disease and habits such as smoking to periodontal disease. ${ }^{1}$ There is also a growing body of evidence linking stress to periodontal disease; however, little has been done to assess the mechanism by which psychosocial stress, distress, and coping influence periodontal disease initiation and progression. The purpose of this paper is to critically evaluate the evidence for an association between stress and periodontal disease and to present models for assessing the mechanisms by which stress may affect periodontal disease.

\section{EVIDENCE FOR THE ASSOCIATION BETWEEN PSYCHOSOCIAL STRESS AND PERIODONTAL DISEASES}

\section{Acute Necrotizing Ulcerative Gingivitis}

Early studies of psychosocial stress and periodontal disease linked acute necrotizing ulcerative gingivitis (ANUG) with stress (for review, see Murayama et al. ${ }^{11}$ ). It has been observed, for example, that there is an increased incidence of ANUG cases in college students during examination periods in comparison to other times. ${ }^{12-14}$ Other studies have also related emotional stress as a predisposing factor to ANUG. ${ }^{15-17} \mathrm{HIV}$ infection has been shown to be associated with severe necrotizing ulcerative gingivitis (NUG), and it has been proposed that stress is responsible at least in part for the increased severity and prevalence of NUG in HIV patients. ${ }^{18-20}$

\section{THE ROLE OF PSYCHOSOCIAL STRESS IN ADULT PERIODONTITIS}

The role of psychosocial disturbances in the etiology of periodontal disease was proposed by many authors in the early literature. ${ }^{21-25}$ Early case-control studies comparing psychiatric patients to controls ${ }^{26}$ found correlations between periodontal disease and age, maladjustment, and hysteria scores, and some measures of psychosomatic disorders. Belting and Gupta ${ }^{27}$ compared 104 outpatient psychiatric cases to 122 outpatients without psychiatric problems at a veterans hospital. Using the Russell Periodontal Index, they found that periodontal disease was more severe in the psychiatric patients as com- 
pared to controls when oral hygiene frequency, level of calculus, age, and bruxism and clenching were held constant. In a study of periodontal disease as related to significant life events, Green and coworkers ${ }^{28}$ assessed 50 subjects, aged 23 to 74 , who were outpatients at a VA hospital dental clinic and required either emergency dental treatment or routine examination. They administered the Life Experiences Survey, which represents life changes that frequently occur in the general population. They also rated the self-reported impact of each event from negative to positive. Periodontal disease was broadly classified as normal, mild, moderate, or severe. The authors also assessed the somatization subscale of the Brief Symptom Inventory. The authors reported significant associations between life event stress and periodontal status. It is not clear from this uncontrolled study to what extent other factors such as age, education, smoking status, and oral cleanliness had on these relationships.

These early studies point to an association between psychosocial stress and distress, but unfortunately do not account for other contributing factors that we know today are likely major risk factors for periodontal disease, such as diabetes mellitus and cigarette smoking. Furthermore, the early studies did not account for coping behaviors and other potential modifiers of the stress response. The above case series and case-control studies are also limited in that they are based upon small samples and subjects who may not be representative of the general population.

Early epidemiological studies, however, do point to a link between psychosocial factors and oral diseases. For example, Osterberg and coworkers ${ }^{29}$ found differences in the oral health status between married and unmarried individuals, and Hunt and coworkers $^{30}$ showed that married people consistently had lower rates of edentulism than unmarried people. More recently, a study by Marcenes and Sheiham ${ }^{31}$ assessed 164 male workers, aged 35 to 44 , measuring work characteristics related to stress. They also measured age, socioeconomic status, sugar consumption, toothbrushing frequency, type of toothpaste, and marital quality. They found that work-related mental demand and marital quality were statistically significantly related to periodontal disease after taking into account the other variables assessed in the study. However, it is not clear whether the authors took into account diabetes and smoking. Smoking, in particular, may have been more common among those under psychosocial stress which could account, in part at least, for the more severe periodontal disease seen in this group.

A later case-control study from this same group $^{32}$ assessed the role of life events in periodontitis. In this study, life events, tobacco use, oral health behaviors, and sociodemographics were assessed. The association between periodontitis and the impact of life events, levels of dental plaque, and employment status remained statistically significant after adjusting for the other variables including smoking. However, tobacco smoking did not remain statistically significantly related to periodontal disease in this model. Marital status became statistically significant after adjusting for all of the other variables. They further showed that the negative impact of life events was related to tobacco smoking, as was being unemployed. Being unemployed was associated with high levels of dental plaque, as well as with tobacco smoking. These findings suggest that life events are associated with periodontal disease either by impacting upon physiologic processes and/or by affecting atrisk behaviors such as smoking and poor oral hygiene, leading to increased disease susceptibility. The association with marital status corroborated previous epidemiological studies that reported differences in oral health status between married and unmarried individuals, ${ }^{29,30}$ suggesting that social support systems also may be an important determinant of oral health.

\section{RESULTS FROM THE ERIE COUNTY EPIDEMIOLOGICAL ASSESSMENT OF STRESS AS A POTENTIAL RISK FACTOR IN PERIODONTAL DISEASE}

The association between psychosocial stress, measures of distress, and coping be- 
haviors was investigated in an epidemiological study of 1,426 participants in the Erie County risk factor study (for a description of the population and methods, see Grossi et al. ${ }^{2,3}$ ). Self-administered questionnaires were used to assess psychosocial stress, measures of psychological distress, and coping style. To estimate stress, the Life Events Scale $^{33}$ and the Daily Strain Scale based on the study of Pearlin and Schooler ${ }^{34}$ were used. Impact and control of life events were also assessed for the Life Events Scale. The strains measured in the Daily Strain Scale were assessed in 5 subscales that include job strain, financial strain, spouse strain, strain of being single, and strain related to parenting children, as well as a global domain termed role strain. Participants in the study were asked to indicate the extent to which they agree with a statement or how frequently conditions occur in each of the 5 social domains. Responses were coded as 1 to 4 , and the average score was calculated for each domain for each participant. Subjects were asked to respond only to items which applied to them; i.e., those subjects who were unemployed at the time of the survey were requested to skip the questions related to job strain, and subjects who were married or dating were asked to skip questions related to the strain of being single.

To measure distress, the Brief Symptom Inventory, an abbreviated version of the SLC90-R, was used to measure psychological distress. ${ }^{35}$ Subjects were asked to rate how much discomfort was experienced over the past week for each of 53 items on a 5-point scale. The Brief Symptom Inventory assessed psychological symptoms in 9 areas: anxiety, somatization, interpersonal sensitivity, psychoticism, paranoid ideation, depression, hostility, phobic anxiety, and obsessive-compulsive. Each symptom is scored on the basis of responses to 4 to 7 items related to that symptom. Anxiety reflects feelings of fear, nervousness, and restlessness; somatization reflects distress arising from bodily complaints such as dizziness, hot spells, and weakness; interpersonal sensitivity addresses problems associated with self-image such as feeling inferior and being easily hurt; and psychoticism can reflect severe psychotic thought patterns, although the authors feel this is more a measure of alienation. ${ }^{36}$ Paranoid ideation reflects a high degree of suspiciousness; depression reflects feelings of loneliness and worthlessness; and hostility reflects a tendency towards confrontation. Phobic anxiety reflects fears and uneasiness associated with unfamiliar surroundings, and obsessive-compulsive reflects cognitive functioning difficulties, such as trouble remembering things and difficulty making decisions.

Coping style was assessed by using the COPE Inventory. ${ }^{37}$ This instrument measures the response of individuals to stress and provides information on problem-focused coping and emotion-focused coping, as well as bad coping. Those who score high on the problem-focused coping scale generally respond to stressful situations by taking action, which can be described as a coping style aimed at solving problems as they arise. Emotion-focused coping is aimed at reducing or managing the emotional stress associated with a disturbing situation. Emotion-focused coping tends to predominate when a situation must be endured.

After participants completed an informed consent form, medical screening questionnaire, and psychosocial questionnaires described above, they underwent a clinical examination. For each tooth present, calibrated examiners performed assessments of supragingival plaque, gingival bleeding, subgingival calculus, probing depth, and clinical attachment level. Probing depth and clinical attachment level were measured with the use of a constant-force electronic periodontal probe equipped with a computerized measurement recording system. In each individual, mean values were calculated from the tooth-specific values, which have been described and the error reported previously. ${ }^{38}$ In addition, alveolar crestal height measurements were made from standardized radiographs according to the technique of Hausmann and Allen. ${ }^{39}$

Screening for specific microorganisms was performed by collection of subgingival plaque and semiquantitative immunofluorescence. Two pooled subgingival plaque samples were collected for each participant. 
Each pooled sample was made up of 6 mesiobuccal samples collected with paper points; one pooled sample came from 6 maxillary teeth and one from 6 mandibular teeth. Using immunofluorescence, the following microorganisms were assessed: $A$. actinomycetemcomitans, Bacteroides forsythus, Campylobacter rectus, Capnocytophaga species, Eubacterium saburreum, Fusobacterium nucleatum, $P$. gingivalis, and Prevotella intermedia.

Venous blood samples were obtained from each subject, and standard blood chemistry measurements such as total cholesterol, albumin, glucose, and uric acid levels were determined. Antibody assays were also performed for 3 putative periodontal disease pathogens: $A$. actinomycetemcomitans, $P$. gingivalis, and $B$. forsythus.

Various cutoffs for defining exposure were initially evaluated. The description of the questionnaire-based responses to the psychosocial variables by case status is presented in Moss et al. ${ }^{40}$ For this initial exploratory case-control analysis of psychosocial factors in periodontitis, we selected 71 cases and 77 controls among the participants $^{40}$ and defined cutoffs for exposures determined from the distribution of these variables in the population. The preliminary exploratory analysis showed that there is likely an association for the global measure, role strain, and periodontal disease that is not completely explained by other factors such as smoking, plaque score, or antibody levels. There was also a strong association between levels of $B$. forsythus and periodontal disease among individuals with a high depression score but not among individuals with low depression scores, suggesting a psychoneuroimmunologic link between $B$. forsythus antibody levels and adult periodontitis. In a longitudinal follow-up of these same cases and controls, it was found that individuals who had elevated levels of antibody to $B$. forsythus and high scores on the depression scale were also more likely to experience periodontal disease. This achieved statistical significance when income level and current smoking status were included as covariates.
We next analyzed the full data from the study of all 1,426 participants in the Erie County risk factor study. ${ }^{2,3}$ In a detailed analysis of stress, distress, and coping behaviors and their association with periodontal disease, we first assessed the reliability of subjects' responses and internal consistency between items and subscales of the psychosocial instruments (unpublished data). Internal consistencies of all of the subscales of each of the instruments used were high, with Cronbach's alpha ranging from 0.88 for financial strain to 0.99 for job strain. Factor analysis also revealed that items on each subscale clustered appropriately for our population.

We next assessed the associations between psychosocial factors and periodontal disease status and found that individuals with high levels of clinical attachment loss had higher scores on the financial strain scales than periodontally healthy individuals. The mean attachment loss was $1.98 \pm$ $0.07 \mathrm{~mm}$ for those with high financial strain versus $1.66 \pm 0.9 \mathrm{~mm}$ for individuals reporting low levels of financial strain, after adjusting for age, gender, and cigarette smoking $(P=0.008)$. A similar significant difference was found for individuals with high levels of financial strain and greater loss of alveolar bone height as compared to the low financial strain group. Stepwise ordinal logistic regression analysis was carried out and showed that of all the measures of stress including life events and daily strains, only financial strain was significantly associated with greater attachment loss (OR 1.70; 95\% CI, 1.09-2.65) and alveolar bone loss (OR 1.68; 95\% CI, 1.20-2.37) after adjusting for age, gender, and cigarette smoking. Also, depression was related to greater attachment loss (OR 1.51; 95\% CI, 1.022.22).

When coping behaviors were evaluated for those with high financial strain, it was found that those who were high emotion-focused copers, a form of inadequate coping, had a higher risk of more severe attachment loss (OR 2.24; 95\% CI, 1.15-4.38) and alveolar bone loss (OR 1.91; 95\% CI, 1.15-3.17) as compared to those with low levels of financial strain within the same coping group. 
These results were adjusted for age, gender, and cigarette smoking. Similar results were found among the low problem-focused copers for attachment loss (OR 2.21; 95\% CI, 1.11-4.38) and alveolar bone loss (OR 2.12; 95\% CI, 1.28-3.51). However, subjects with high levels of financial strain who reported high levels of problem-focused coping, a form of adequate or good coping, had no more periodontal disease than those with low levels of financial strain. These analyses suggest that the effects of stress on periodontal disease can be moderated by adequate coping behavior. In addition, subjects with higher financial strain and those with depression had significantly more risk for periodontal disease after adjusting for age; gender; cigarette smoking; systemic diseases such as diabetes mellitus, allergy, and anemia; periodontal microorganisms; and emotion-focused coping.

In summary, psychosocial measures of stress associated with financial strain and distress (manifested as depression) are significant risk indicators for periodontal disease in adults. Of considerable interest is the observation that adequate coping behaviors as evidenced by high levels of problemfocused coping may reduce the stress-associated risk for periodontal disease.

Whether stress-associated risk for periodontal disease is related to psychoneuroimmunologic changes and/or behavioral changes is yet to be determined. However, one indication that psychoneuroimmunologic mechanisms may play a role comes from our assessment of salivary cortisol in the Erie County study (unpublished data). We evaluated a randomly selected group of patients from the Erie County study who reported high levels of financial strain and poor coping strategies and found that they had significantly higher levels of salivary cortisol as compared to a control group who exhibited no significant attachment loss, had low financial strain, and were low emotion-focused copers. Salivary samples were also adjusted for Circadian variation in cortisol levels by matching the time of day at which the samples were collected. Salivary cortisol levels, rather than serum levels, were selected since taking saliva is not subject to alterations associated with the stress of venipuncture. There was a direct correlation of salivary cortisol levels and alveolar bone loss in patients with established periodontitis who had high levels of financial strain and poor coping strategies. The partial correlation coefficient between alveolar bone loss and salivary cortisol level in this group was 0.71 after adjusting for gender, age, smoking (pack years), financial strain, and emotion-focused coping. These associations of salivary cortisol levels and stress, coping, and periodontal disease suggest that the effect of stress on periodontal disease is mediated, at least in part, by psychoneuroimmunologic mechanisms.

\section{MOLECULAR AND ENDOCRINE MECHANISMS OF THE STRESS RESPONSE}

The cellular and molecular basis for the interaction between the immune system and central nervous system (CNS), which mediates the effects of stress in maintaining immunologic and behavioral homostasis, has been the subject of intense study. Analyses of the role of stress in infectious processes such as periodontal disease should consider the overall effect of stressors appraised by the brain. The stress-induced responses either result in change in behavior or are transmitted to the hypothalamic/pituitary/ adrenal (HPA) axis to promote the release of corticotropic-releasing hormone (CRH) from the hypothalamus and glucocorticoids from the adrenal cortex (Fig. 1). Stress perceived by the brain stimulates the hypothalamus to produce $\mathrm{CRH}$, which is released into the hypophyseal portal system, activating the pituitary gland to release adrenocorticotropic hormone $(\mathrm{ACTH})$, which in turn induces release of corticosteroids from the adrenal cortex.

Glucocorticoids, including cortisol (the primary glucocorticoid), exert major suppressive effects through highly specific mechanisms at multiple levels. For example, in vivo glucocorticoids reduce the number of circulating lymphocytes, monocytes, and eosinophils. They also inhibit the accumulation of eosinophils, macrophages, and neutrophils at inflammatory sites. ${ }^{41,42}$ At the 


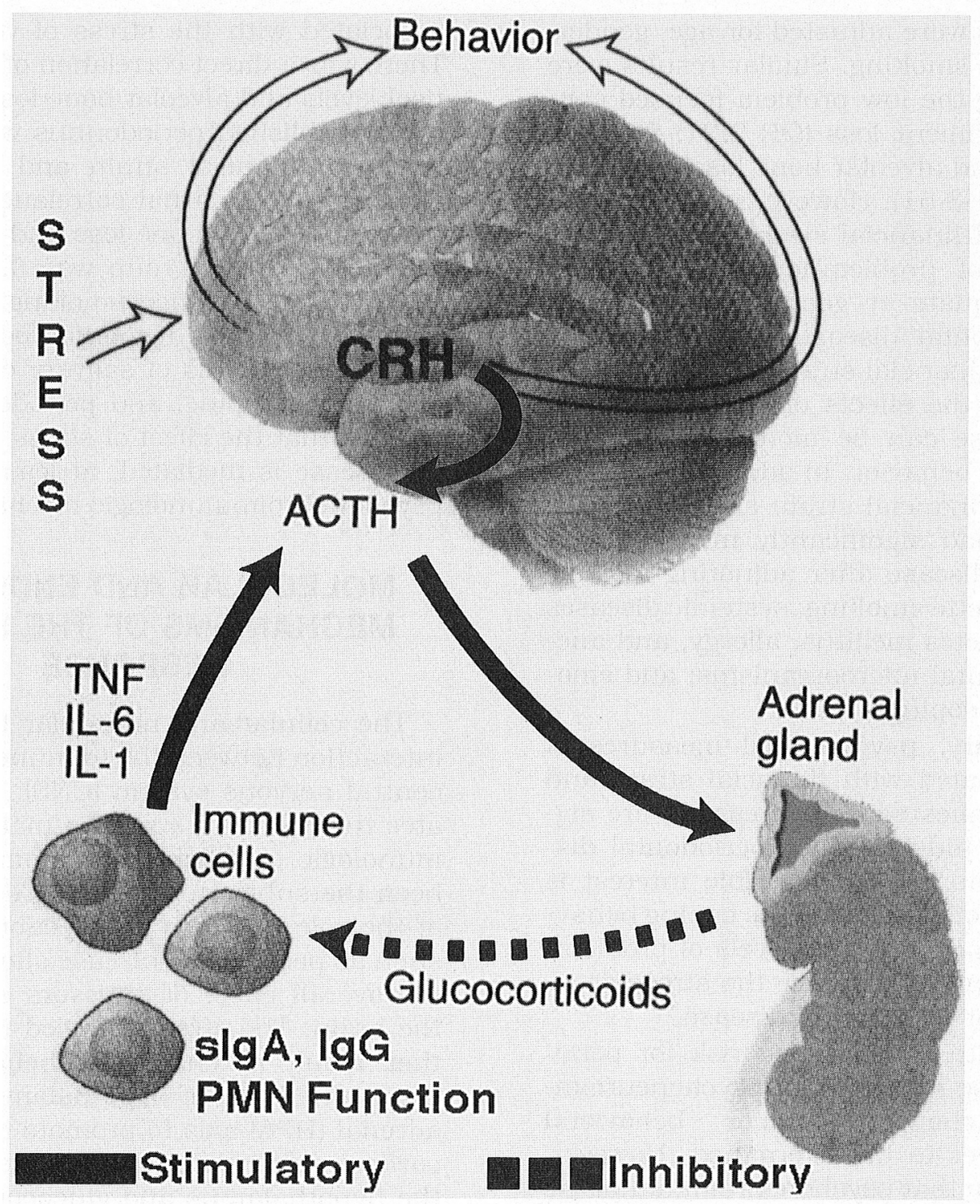

Figure 1. Behavioral and physiological effects of stress. $\mathrm{CRH}=$ corticotropic-releasing hormone; $\mathrm{ACTH}=$ adrenocorticotropic hormone; TNF = tumor necrosis factor; $\operatorname{slg} \mathrm{A}=$ secretory immunogiobulin A. Modified with permission from the Annals of Internal Medicine 1992;117: 855.

molecular level, glucocorticoids profoundly inhibit important functions of inflammatory cells including macrophages, neutrophils, eosinophils, and mast cells in functions such as chemotaxis, secretion, and degranulation. ${ }^{42,43}$ Glucocorticoids also inhibit the cascade of the immune response by inhibiting macrophage-antigen presentation, lymphocyte proliferation, and lymphocyte differentiation to effector cell types such as helper lymphocytes, cytotoxic lymphocytes, natural killer cells, and antibody-forming $B$ cells. ${ }^{44}$ Corticosteroids also inhibit production of cytokines including IL-1, IL-2, IL-3, and IL-6, tumor necrosis factor, interferon gamma, and granulocyte and monocyte colony stimulating factors. Glucocorticoids inhibit arachidonic acid-derived proinflammatory mediators such as prostaglandins and leukotrienes. ${ }^{45}$ Glucocorticoids also in- 
duce endogenous anti-inflammatory proteins and lipocortins, which have the capability of inhibiting phospholipase A2, thereby inhibiting generation of eicosanoids. ${ }^{43}$ Hence, the stress-related stimulation of the HPA axis with the production of glucocorticoids such as cortisol has major suppressive actions on immune and inflammatory responses. This represents the major effector arm of the CNS-hormonal axis.

There is also an afferent or feedback arm consisting of stimulation of the HPA axis by cytokines (Fig. 1). Although physical and emotional stressors are major stimulators of the HPA axis, soluble molecules of the immune system such as the cytokines can also activate this axis. These peripherally generated cytokines activate the HPA axis at all of its levels including hypothalamic CRH neuron, pituitary corticotrophs, as well as the adrenal cortex. ${ }^{46-49}$ Peripherally generated inflammatory mediators that can activate the HPA axis include lymphocyte-derived gamma interferon, IL-2, IL-6, macrophage-derived IL1, and tumor necrosis factor (Fig. 1).

The infrastructure for communication between the central nervous system and immune system which acts as a feedback loop is beginning to be understood at the molecular and cellular level. This psychoneuroimmunologic network may explain the role of stress in infections and inflammatory diseases of man. ${ }^{50}$

\section{MENTAL STRESS RESPONSE TRIGGERING THE HPA AXIS WITH IMMUNOSUPPRESSIVE EFFECTS: A MODEL FOR UNDERSTANDING THE ROLE OF STRESS IN PERIODONTAL DISEASE}

One model to evaluate the role of psychosocial stress and coping behaviors in periodontal disease is depicted in Figure 2 . According to this model, psychosocial stress can activate the central nervous system. The hypothalamus releases CRH which, among other things, stimulates release of ACTH from the pituitary, which in turn results in production of cortisol by the adrenal cortex. Glucocorticosteroids, including cortisol, then depress immunity including secretory IgA, IgG, and neutrophil functions, all of which may be important in protection against infection by periodontal organisms. Secretory IgA antibodies may protect by reducing initial colonization of periodontal pathogens. IgG antibodies may exert protection by opsonizing periodontal organisms for phagocytosis and killing by neutrophils. This then gives rise to increased susceptibility, which leads to the establishment of periodontal infection which, in turn, results in destructive periodontitis. Periodontitis is brought about by tissue-destroying factors such as IL-1 and matrix metalloproteinases activated by the periodontal pathogens, as well as by the direct effects of pathogenic bacteria.

Evaluation of salivary IgA levels in patients with periodontal disease as compared to normal subjects has been controversial. For example, Brandtzaeg et al. ${ }^{51}$ found that IgA concentration in whole saliva is higher in subjects with periodontal inflammation than in clinically healthy individuals. A study by Chandler et al. ${ }^{52}$ failed to demonstrate differences in parotid salivary IgA concentration or in secretion rate when correlated to periodontal inflammation. However, the latter study was small, with only 12 subjects free of oral infection as compared to 40 subjects with varying levels of periodontal disease. Clearly, levels of parotid serum antibodies and other immunologic functions in patients with periodontal disease who are under stress need to be evaluated to assess whether model 1 is accurate in describing the psychoneuroimmunologic events occurring in periodontal disease in individuals under stress.

Mental as well as physical stress can also result in responses being transmitted to the autonomic nervous system and then to the adrenal medulla, resulting in secretion of catecholamines such as epinephrine and norepinephrine. ${ }^{53,54}$ Catecholamines then affect prostaglandin and proteases, which in turn, could enhance periodontal destruction.

Evidence for model 1 is limited and consists of reports of altered salivary IgA in periodontitis patients, ${ }^{51}$ along with our findings of elevated salivary cortisol levels in subjects 


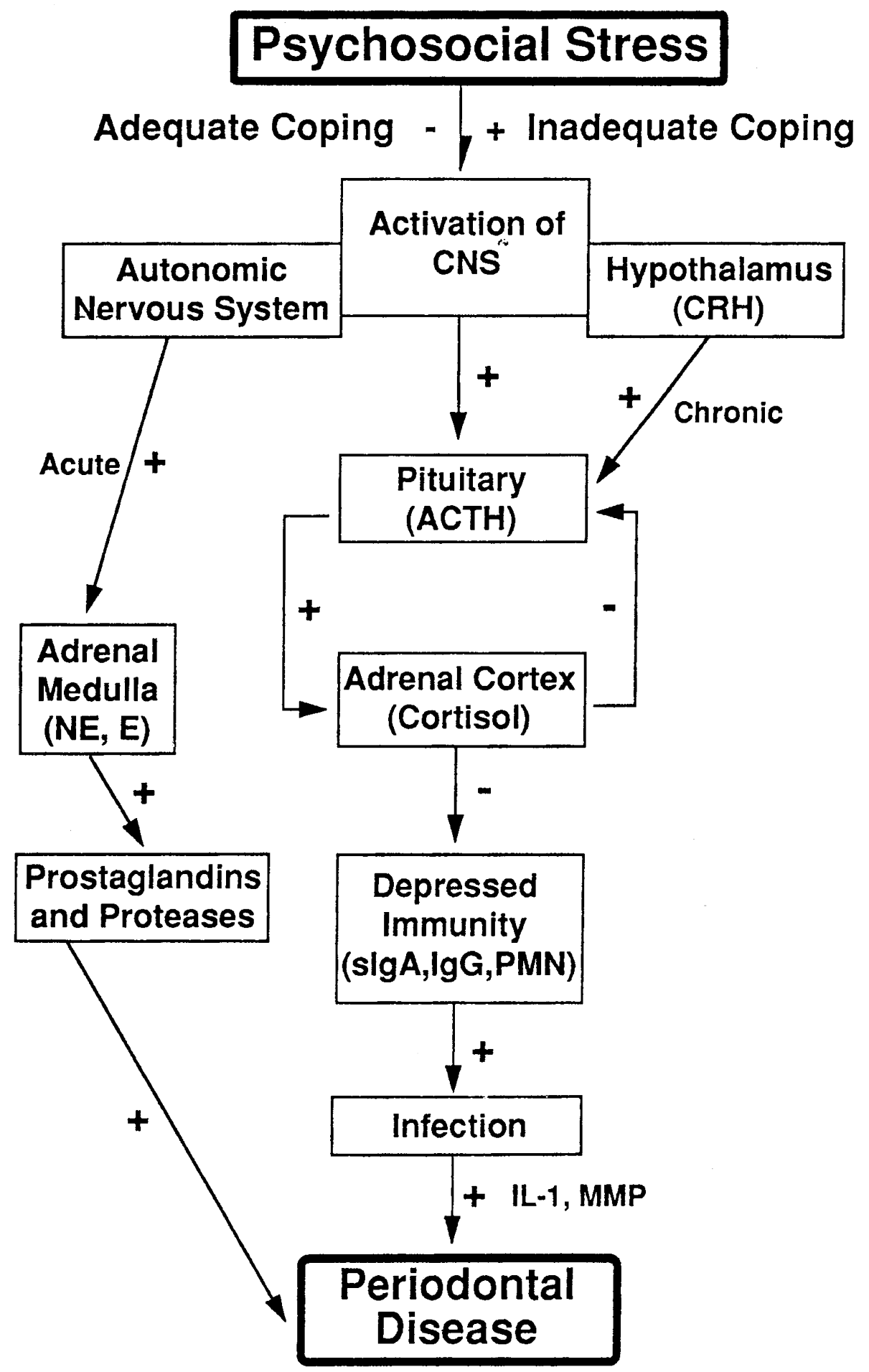

Figure 2. Model 1. A physiological model for effects of stress on periodontal disease. CNS = central nervous system; $\mathrm{CRH}=$ corticotropic-releasing hormone; $\mathrm{ACTH}=$ adrenocorticotropic hormone; $\mathrm{NE}=$ norepinephrine; $\mathrm{E}=$ epinephrine; $\mathrm{MMP}=$ matrix metalloproteinase. 


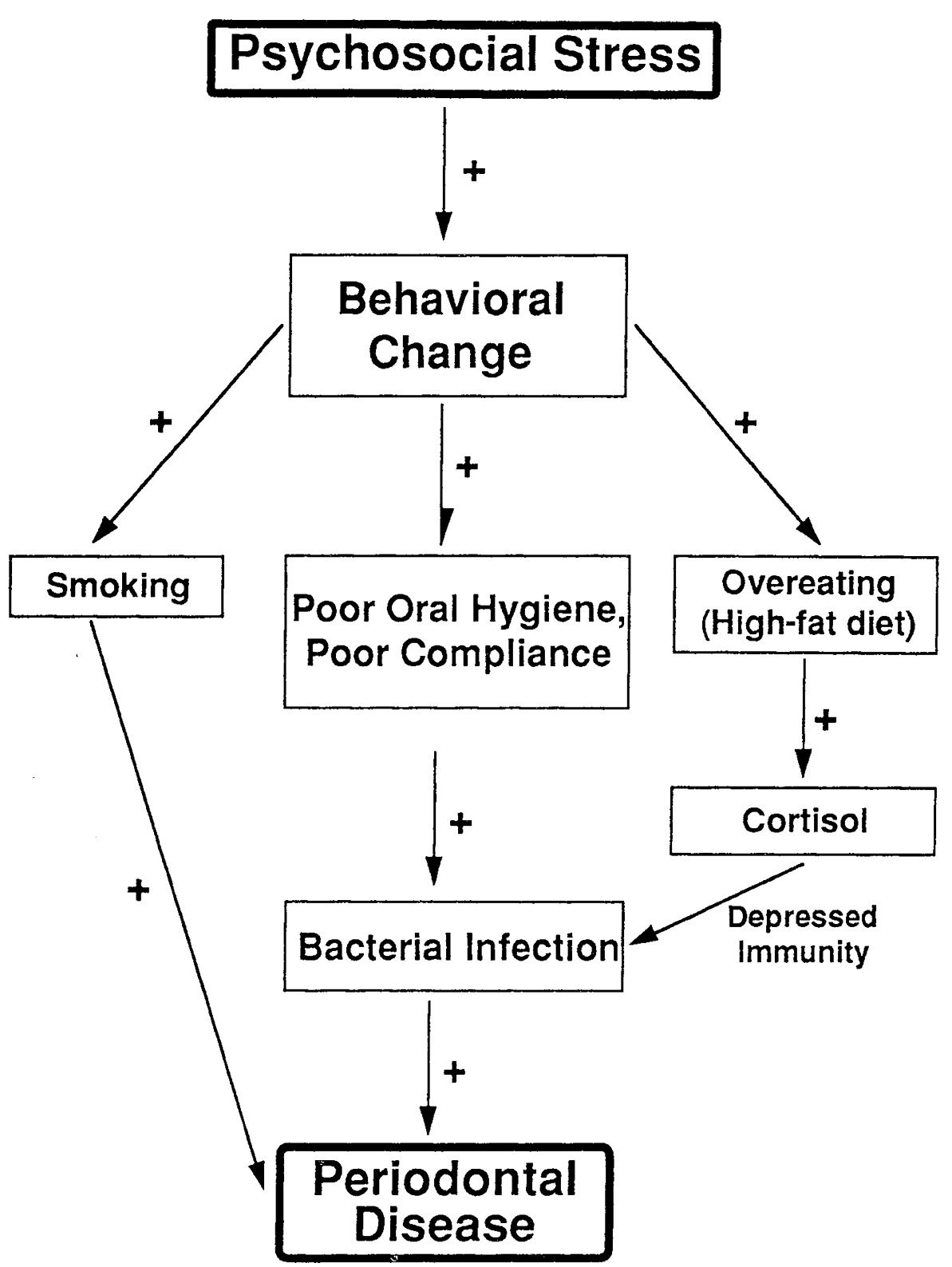

Figure 3. Model 2. Psychosocial stress and its effect on behavior as manifested by alterations in periodontal disease.

with severe periodontal disease who have financial strain and are inadequate copers. To fully evaluate model 1 , experiments must be expanded to other populations. In addition, they must assess immune functions and include longitudinal studies in which the time sequence of elevated cortisol levels and immune dysfunction and development of periodontal diseases are evaluated.

\section{MENTAL STRESS RESPONSE LEADING TO BEHAVIORAL CHANGES}

In the second model of the role of psychosocial stress on periodontal disease, it is hypothesized that the main effects of stress occur through behavioral changes which affect at-risk health behaviors such as smoking, poor oral hygiene, and poor compliance 
with dental care (Fig. 3). There is also a possibility that stress leads to other behavioral changes such as overeating, especially a high-fat diet, which then can lead to immunosuppression through increased cortisol production. There are certainly many other possible behaviors that could be affected by stress and inadequate coping and distress, such as depression, which would have significant effects on periodontal disease. Any evaluation of the role of stress in periodontal disease would have to take into consideration these at-risk health care behaviors to determine to what extent they contribute to the interaction between stress and periodontal disease.

\section{STRESS HYPOTHESIZED AS A COMMON PATHWAY FOR SEVERAL RELATED CHRONIC DISEASES OF MAN}

It has become clear that not only do systemic diseases such as diabetes (see review by $\mathrm{Genco}^{1}$ ) and osteoporosis ${ }^{7}$ increase the risk for periodontal disease, but also that periodontal disease may increase the risk of systemic diseases such as cardiovascular disease. ${ }^{55-58}$ Periodontal disease may also increase the risk for preterm delivery. ${ }^{59}$

It is well established that cardiovascular disease, diabetes mellitus, preterm delivery, and osteoporosis are related to stress either as a physiological response to stress or as a behavioral response. It may be that stress is a significant common risk factor for diabetes mellitus, cardiovascular disease, preterm delivery, and osteoporosis, as well as periodontal disease (Fig. 4). Of course, different stressors and different responses to stress may be operative in each disease.

Communication between the central nervous system and the immune and endocrine systems has given rise to the field of psychoneuroimmunology. For example, Ader and Cohen $^{60}$ showed that the central nervous system is involved in the immune response. The role of the HPA axis and cytokine regulatory loop as described above and depicted graphically in Figure 1 is reasonably well established. However, it is becoming clear that stress can also regulate neuroendocrine functions. ${ }^{61}$ For example, when the hypothalamic-pituitary-gonadal (HPG) axis is called into play or inhibited during stress, there is production of sex hormones such as estrogen, progesterone, prolactin, and growth hormone which have major effects on the immune system, and alpha-melanocytestimulating hormone which is anti-inflammatory. ${ }^{62-66}$ These factors may be involved in gender-associated conditions such as postmenopausal osteoporosis.

Accordingly, we hypothesize that stress is a common pathway for several chronic diseases of man. Psychosocial stress as modified by perceptions and coping can lead to physiological processes through: 1) the HPA axis, resulting in glucocorticosteroids; 2) the autonomic nervous system, resulting in the release of catecholamines; or 3) the HPG axis, resulting in the release of sex hormones. These could have major effects on cardiovascular disease, diabetes, periodontal disease, preterm delivery, and osteoporosis. Alternately or simultaneously, stress that is modified by perceptions in coping can give rise to at-risk health behaviors, which then could affect the same spectrum of chronic diseases.

It is clear that much is to be learned about the role of stress in these diseases and that if stress is indeed a common causal pathway, to what extent intervention in stress will have an effect in moderating these major chronic, debilitating diseases of man. Studies of risk factors for periodontal disease, diabetes, cardiovascular disease, preterm delivery, and osteopenia should include a rigorous evaluation of stress, distress, and coping behaviors for a full understanding of the risk patterns among these diseases.

\section{GUIDELINES FOR EVALUATION OF THE ROLE OF PSYCHOSOCIAL STRESS IN PERIODONTAL DISEASE}

We propose guidelines for the evaluation of the role of psychosocial stress in periodontal disease for future studies. As we learn more about this field, other guidelines and experimental approaches may also be useful. 


\section{Stress hypothesized as a common pathway for several chronic diseases of man}

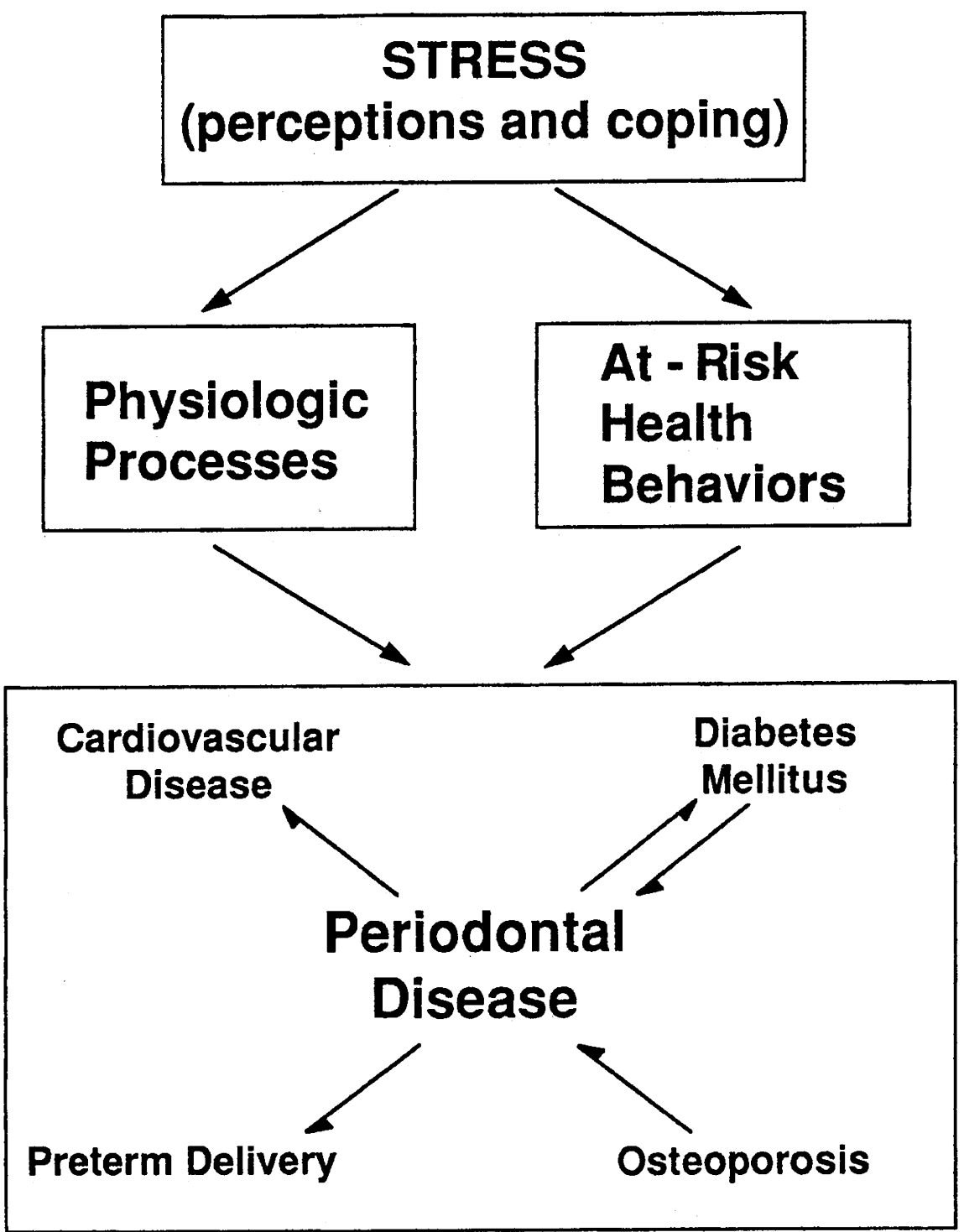

Figure 4. The common pathway of stress related to periodontal disease, cardiovascular disease, diabetes mellitus, preterm delivery, and osteoporosis.

1. Periodontal disease should be measured as a unique disease outcome, not included in a composite index with other oral diseases.

2. Stress and distress, as well as coping behaviors, should each be assessed by validated instruments. These instruments, of course, would be best validated by prior study but also should be validated on the particular population to which they are applied.

3. At-risk behaviors should be measured including oral health behaviors such as preventive dental visits, compliance with oral hygiene regimens, and an assessment of plaque, gingivitis, and other existing dis- 
ease(s) as this may indicate compliance with oral health regimens.

4. Case series and case-control studies can generate hypotheses; however, large cross-sectional and longitudinal epidemiological studies may be needed to rigorously establish that psychosocial factors such as stress, distress, and coping behaviors are indeed true risk factors for periodontal disease.

5. Studies of mechanism(s) by which psychosocial stress or distress exert effects on periodontal disease are needed to establish the biological rationale for this relationship. Such studies may include assessment of biochemical mediators of stress, immune functions, or neurological and endocrine alterations, as well as behavioral changes. Animal models to test mechanisms may be instructive.

6. Intervention studies using stress management to reduce stress or distress with randomized controlled trial methodology are necessary to establish efficacy of modification of stress as a modality in the management of stress-associated disease.

\section{SUMMARY}

Studies to date strongly suggest that stress, distress, and inadequate coping are important risk indicators for periodontal disease. Furthermore, it is likely that systemic diseases associated with periodontal disease such as diabetes, cardiovascular disease, preterm delivery, and osteoporosis may share psychosocial stress as a common risk factor. These early beginnings will require extensive study to fully understand the molecular and cellular basis of the role of stress, and in turn these studies may lead to effective intervention strategies that minimize or negate stress as a contributor to periodontal diseases.

\section{Acknowledgments}

The authors are grateful to the staff of the University at Buffalo Periodontal Disease Research Center, including Drs. Ernest Hausmann and Joseph Zambon; and Mrs. Yolande Gratton and Mrs. Cindy Nowadly for their efforts in carrying out the Erie County Epide- miologic Study described here. The critical discussions of the data on stress and coping by Drs. James Beck and Mark Moss were very much appreciated. The insights of the global role of cortisol by Dr. Eugene Emory in a wide spectrum of diseases including preterm delivery are also appreciated. These studies were supported in part by the USPHS National Institutes of Health grant DE04898, Unilever Research, and Sunstar Inc.

\section{REFERENCES}

1. Genco RJ. Current view of risk factors for periodontal diseases. J Periodontol 1996;67(suppl.):10411049.

2. Grossi SG, Zambon JJ, Ho AW, et al. Assessment of risk for periodontal disease. I. Risk indicators for attachment loss. J Periodontol 1994;65:260-267.

3. Grossi SG, Genco RJ, Machtei EE, et al. Assessment of risk for periodontal disease. II. Risk indicators for alveolar bone loss. $J$ Periodontol 1995;66:23-29.

4. Oliver RC, Brown LJ, Löe H. Variations in the prevalence and extent of periodontitis. J Am Dent Assoc $1991 ; 122: 43-48$.

5. Robinson P. Periodontal disease and HIV infection. Review of the literature. $J$ Clin Periodontol 1992;19: 609-614.

6. Robinson PB, Sheiham A, Challacomb SJ, Zakrzewska JM. The periodontal health of homosexual men with HIV infection. A controlled study. Oral Dis 1996;2:45-52.

7. Wactawski-Wende J, Grossi SG, Trevisan M, et al. The role of osteopenia in oral bone loss and periodontal disease. J Periodontol 1996;67(suppl.): 10761084.

8. Daniel MA, Van Dyke TE. Alterations in phagocyte function and periodontal infection. $J$ Periodontol 1996;67(suppl.):1070-1075.

9. Wilson ME, Kalmar JR. Fc $\gamma$ RIIa (CD32): A potential marker defining susceptibility to localized juvenile periodontitis. J Periodontol 1996;67:323-331.

10. Kornman KS, Crane A, Wang H-Y, et al. The interleukin-1 genotype as a severity factor in adult periodontal disease. J Clin Periodontol 1997;24:72-77.

11. Murayama Y, Kurihara H, Nagai A, Dompkowski D, Van Dyke TE. Acute necrotizing ulcerative gingivitis: Risk factors involving host defense mechanisms. Periodontol 2000 1994;6:116-124.

12. Kur DA. Gingival and periodontal disease. $J A m$ Dent Assoc 1945;32:31-33.

13. Giddon DB, Goldhaber P, Dunning JM. Prevalence of reported cases of acute necrotizing ulcerative gingivitis in a university population. $J$ Periodontol 1963;34:66-70. 
14. Giddon DB, Zackin SJ, Goldhaber P. Acute necrotizing ulcerative gingivitis in college students. $J \mathrm{Am}$ Dent Assoc 1964;68:381-386.

15. Cohen-Cole SA, Cogen RB, Stevens AW Jr, et al. Psychiatric, psychological, and endocrine correlates of acute necrotizing ulcerative gingivitis. Psychiatr Med 1983;1:215-225.

16. Shore RS, Havrilla J. Acute necrotizing ulcerative gingivitis: Etiology and stress relationships. Int $J$ Psychosom 1986;83:35-40.

17. Melnick SL, Roseman JM, Engel JD, et al. Epidemiology of acute necrotizing ulcerative gingivitis. Epidemiol Rev 1988;10:191-211.

18. Schiott M, Pindborg JJ. AIDS and the oral cavity: Epidemiology and clinical oral manifestations of human immune virus infection. A review. Int $J$ Oral Maxillofac Surg 1987;16:1-14.

19. Winkler JR, Robertson PB. Periodontal disease associated with HIV infection. Oral Surg Oral Med Oral Pathol 1992;73:145-150.

20. Horning GM, Cohen ME. Necrotizing ulcerative gingivitis, periodontitis, and stomatitis: Clinical staging and predisposing factors. $J$ Periodontol 1995;66: 990-998.

21. Burstone NS. The psychosomatic aspects of dental problems. J Am Dent Assoc 1946;33:862-871.

22. Weller CV. Constitutional factors in periodontitis. $J$ Am Dent Assoc 1928;15:1081-1086.

23. Miller SC, Firestone JM. Psychosomatic factors in the etiology of periodontal disease. Am J Orthodont Oral Surg 1947;33:675-686.

24. Moulton R, Ewen S, Thieman W. Emotional factors in periodontal disease. Oral Surg Oral Med Oral Pathol 1952;5:833-860.

25. Tervonen T, Knuuttila M, Nieminen P. Risk factors associated with abundant dental caries and periodontal pocketing. Community Dent Oral Epidemiol 1991;19:82-87.

26. Baker EG, Crook GH, Schwacher ED. Personality correlates of periodontal diseases. J Dent Res 1961; 40:396-403.

27. Belting CM, Gupta OP. The influence of psychiatric disturbances on the severity of periodontal disease. $J$ Periodontol 1961;32:219-226.

28. Green LW, Tryon WW, Marks B, Huryn J. Periodontal disease as a function of life events stress. $J \mathrm{Hu}$ man Stress 1986;12:32-36.

29. Osterberg T, Hedegard B, Sater G. Variation in dental health in 70-year-old men and women in Goteborg, Sweden. A cross-sectional epidemiologic study including longitudinal and cohort effects. Swed Dent J 1984;8:29-48.

30. Hunt RJ, Beck JD, Lemke JH, Cohout FJ, Wallace RB. Edentulism and oral health problems among elderly rural Iowans: The Iowa 65+ rural health study. Am J Public Health 1985;75:1177-1181.

31. Marcenes WS, Sheiham A. The relationship between work stress and oral health status. Soc Sci Med 1992;35:1511-1520.

32. Croucher R, Marcenes WS, Torres MCMB, Hughes F, Sheiham A. The relationship between life events and periodontitis. A case control study. $J$ Clin Periodontol 1997;24:39-43.
33. Dowrenwend BS, Drasnoff L, Ashenasj AR, Dowrenwend BP. Exemplification of a method for scaling life events: The PERI life events scale. $J$ Health Soc Behav 1978; 19:205-229.

34. Pearlin LI, Schooler C. The structure of coping. $J$ Health Soc Behav 1978;19:2-21.

35. Derogatis LR, Cleary PA. Confirmation of the dimensional structure of the SCL-90: A study in construct validation. $J$ Clin Psychol 1977;33:981-989.

36. Derogatis LR, Melisaratos N. The Brief Symptom Inventory: An introductory report. Psychol Med 1983; 13:595-605.

37. Carver CS, Scheier MF, Weintraub JK. Assessing coping strategies: A theoretically based approach. $J$ Pers Soc Psychol 1989;56:267-283.

38. Grossi SG, Dunford HG, Ho A, Koch G, Machtei EE, Genco RJ. Sources of error for periodontal probing measurements. $J$ Periodont Res 1996;31:330-336.

39. Hausmann E, Allen K. Reproducibility of bone height measurements made on serial radiographs. $J$ Periodontol 1997;68:839-841.

40. Moss ME, Beck JD, Kaplan BH, et al. Exploratory case-control analysis of psychosocial factors and adult periodontitis. $J$ Periodontol 1996;67(suppl.): 1060-1069.

41. Cupps TR, Fauci AS. Corticosteroid-mediated immunoregulation in man. Immunol Rev 1982;65: 133-155.

42. Schleimer RP. Effects of glucocorticosteroids on inflammatory cells relevant to their therapeutic applications in asthma. Am Rev Respir Dis 1990;141: S59-S69.

43. Schleimer RP, Freeland HS, Peters SP, Brown KE, Derse CP. An assessment of the effects of glucocorticoids on degranulation, chemotaxis, binding to vascular endothelium, and formation of leukotriene-B4 by purified human neutrophils. J Pharmacol Exp Ther 1989;250:598-605.

44. Snyder DS, Unanue ER. Corticosteroids inhibit murine macrophage LA expression and interleukin-1 production. J Immunol 1982;129:1803-1805.

45. Williams TJ, Yarwood H. Effect of glucocorticosteroids on microvascular permeability. Am Rev Respir Dis 1990;141:S39-S43.

46. Woloski BM, Smith EM, Meyer WJ III, Fuller GM, Bialock JE. Corticotropin-releasing activity of monokines. Science 1985;230:1035-1037.

47. Bernton EW, Beach JE, Holaday JW, Smallridge RC, Fein HG. Release of multiple hormones by direct action of interleukin-1 on pituitary cells. Science 1987;238:519-521.

48. Bernardini R, Luger A, Gold PW, Chiarenza A, Legakis J, Chrousos GP. Cytokines stimulate pituitary adrenal function via activation of the CHR neuron. In: F. Petraglia, ed. New Trends in Brain and Female Reproductive Function. Rome: CIG Edizioni Internazionali; 1988.

49. Sternberg EM. Monokines, lymphokines, and the brain. In: Cruse JM, Lewis RE Jr, eds. The Year in Immunology, vol. 5. Basel, Switzerland: Karger; 1988:205-217.

50. Sternberg EM, Licinio J. Overview of neuroimmune stress interactions. Implications for susceptibility 
to inflammatory disease. Ann NY Acad Sci 1995; 771:364-371.

51. Brandtzaeg P, Fjellanger I, Gjeruldsen ST. Human secretory immunoglobulins. I. Salivary secretion of individuals with normal or low levels of serum immunoglobulins. Scand J Haematol 1970; 12(suppl.): 3-83.

52. Chandler DC, Silverman NS, Lundblad RL, McFall WT. Human parotid IgA and periodontal disease. Arch Oral Biol 1974; 19:733-735.

53. Dimsdale JE, Moss J. Plasma catecholamines in stress and exercise. JAMA 1980;243:340-342.

54. Fibiger W, Singer G, Miller A. A relationship between catecholamines in urine and physical and mental effort. Int $J$ Psychophysiol 1984; 1:325-333.

55. Mattila K, Nieminen M, Valtonen V, et al. Association between dental health and acute myocardial infection. Br Med $J$ 1989;298:779-782.

56. Mattila KJ, Valle MS, Nieminen MS, Valtonen VV, Hietaniemi KL. Dental infections and coronary atherosclerosis. Atherosclerosis 1993;103:205-211.

57. DeStefano F, And RF, Kahn HS, Williamson DF, Russell CM. Dental disease and risk of coronary heart disease and mortality. $\mathrm{Br}$ Med $J$ 1993;306: 688-691.

58. Beck J, Garcia R, Heiss G, Vokonas PS, Offenbacher S. Periodontal disease and cardiovascular disease. J Periodontol 1996;67(suppl.):1123-1137.

59. Offenbacher S, Katz V, Fertik G, et al. Periodontal infection as a possible risk factor for perterm low birth weight. J Periodontol 1996;67(suppl.):11031113.
60. Ader R, Cohen N. Psychoneuroimmunology. Orlando, FL: Academic Press; 1981:281.

61. Blalock JE. A molecular basis for bidirectional communication between the immune system and neuroendocrine system. Physiol Rev 1989;69:1-32.

62. Rivier C, Rivier J, Vale W. Stress induced inhibition of reproductive functions: Role of endogenous corticotropin-releasing factor. Science 1986;231:607609.

63. Vernikos J, Dallman MF, Keil LC, O'Hara D, Convertino VA. Gender differences in endocrine responses to posture in seven days of $-6^{\circ}$ head down bed rest. Am J Physiol 1993;265:E153-E161.

64. Wilder RL, Sternberg EM. Neuroendocrine hormonal factors in rheumatoid arthritis and related conditions. Curr Opin Rheumatol 1990;2:436-440.

65. Hiltz ME, Lipton JM. Alpha-MSH peptides inhibit acute inflammation in context sensitivity. Peptides 1990;11:979-982.

66. Cannon JG, Tatro JB, Reichlin S, Dinarello CA. Alpha-melanocyte-stimulating hormone inhibitis immunostimulatory and inflammatory actions of IL-1. $J$ Immunol 1986; 137:2232-2236.

Send reprint requests to: Dr. Robert Genco, State University of New York at Buffalo, Department of Oral Biology, 3435 Main Street, Foster Hall, Buffalo, NY 14214-3092. Fax: 716/829-2387; e-mail: RJGenco@ acsu.buffalo.edu

Accepted for publication February 16, 1998. 